\title{
EstomatoPEP: Prontuário eletrônico desenvolvido para otimizar a educação em serviço numa clínica escola de odontologia
}

\author{
Aline Cachate de Farias- CESMAC- alinecachate@hotmail.com \\ Carlos Alberto Correia Lessa Filho- CESMAC - carloswgama@gmail.com \\ Arthur Eric Costa Wanderley- CESMAC- arthur.ecw@gmail.com \\ Thiago José Gomes Oliveira - CESMAC - thiagooliveira.ic@gmail.com \\ Luciano Bairros- CESMAC - lucianopsico@yahoo.com.br \\ Sonia Maria Soares Ferreira - CESMAC - sonia.ferreira@cesmac.edu.br
}

\begin{abstract}
Resumo: O sistema de Prontuário Eletrônico do paciente (PEP) é um recurso tecnológico que consiste em uma ferramenta muito eficaz. Desta forma, o foco principal desta pesquisa foi desenvolver e validar um PEP voltado para estomatologia na forma de aplicativo para Smartphones e Tablets, tendo como parâmetro o prontuário físico utilizado na triagem de pacientes de uma Clínica Escola de Odontologia. O aplicativo foi desenvolvido usando o framework Ionic e Cordova e por questões de segurança foi desenvolvido um webservice que está alocado dentro da própria instituição. Para a construção do webservice foi utilizado o interpretador NodeJS com framework Express, para recebimento das requisições e respostas do aplicativo. A fase de validação foi realizada através da metodologia de grupo focal e oficinas com professores, alunos e pacientes onde foram testadas todas as funcionalidades e realizados os ajustes até a versão final. A versão final está registrada no Instituto Nacional da propriedade Intelectual. Conclui-se nesse estudo que a implantação do PEP na clínica escola de odontologia trará agilidade, segurança, qualidade de armazenamento de informações e geração de relatórios para as atividades clínicas dando suporte para as pesquisas, trazendo um retorno importante para a comunidade acadêmica e para a sociedade.
\end{abstract}

Palavras-chave: Registros eletrônicos de saúde. Odontologia. Software.

\section{EstomatoPEP: Electronic Medical Record designed to optimize in-service education in a dental school clinic}

Abstract: The Electronic Medical Record (EMR) system is a technological resource that is a very effective tool. Thus, the main focus of this research was to develop and validate a EMR focused on stomatology in the form of application for Smartphones and Tablets, having as a parameter the physical chart used to screen patients in a School of Dentistry Clinic. The application was developed using the Ionic and Cordova framework and for security reasons a webservice has been developed which is allocated within the institution itself. For the construction of webservice, the NodeJS interpreter with framework Express was used to receive requests and responses from the application. The validation phase was performed through the focus group methodology and workshops where the software was used in the clinic with patient groups testing all functionalities and adjustments until the final version. The final version is registered with the National Institute of Intellectual Property. It is concluded in this study that the implementation of EMR in the dental school clinic will bring agility, security, quality of information storage and reporting for clinical activities supporting research, bringing an important return for the academic community and for society.

Keywords: Electronic Health Records. Dentistry. Software 


\section{Introdução}

A elaboração do diagnóstico final de uma determinada patologia envolve várias habilidades e conhecimentos podendo representar para os estudantes e profissionais da odontologia, uma atividade desafiadora. A própria dinâmica do diagnóstico produz uma série de informações que, se não forem tratadas e armazenadas adequadamente, podem dificultar o processo clínico além de tornar mais difíceis, os estudos epidemiológicos e tomadas de decisão (SANTOS; PACHECO, 2010).

$\mathrm{O}$ conjunto de documentos ordenados e concisos consistem em um prontuário. $\mathrm{O}$ prontuário impresso por muito tempo foi o único instrumento de coleta e armazenamento de informações referentes aos pacientes atendidos nos serviços de saúde (GODOY et al., 2011). O prontuário odontológico permite ao cirurgião-dentista comprovar quando e como o diagnóstico e o tratamento dos pacientes foram realizados (SARAIVA, 2011; BRAGA, 2013) como também deve constar ações realizadas pelo profissional no exercício da profissão, podendo ser utilizadas em processos jurídicos ou periciais (SOARES et al., 2006).

A implementação da tecnologia da informação na saúde é um marco significativo para muitas nações ao redor do mundo (DUFTSCHMID et al., 2004) portanto, a possibilidade de trabalhar com arquivos digitais traz inúmeras vantagens (SOARES et al., 2006). Dentre elas, agilidade, praticidade e otimização do tempo de trabalho de vários profissionais (MACHADO, SCHERMA e PISA, 2012). Os prontuários eletrônicos são ferramentas armazenadas em computador projetado para fornecer aos clínicos, dados completos e informações importantes sobre os pacientes (BRAGA et al., 2013). Dentre as vantagens temos o aumento da produtividade e uma maior facilidade de fazer pesquisa em serviços clínicos de saúde.

O presente estudo teve como objetivo desenvolver e validar um Prontuário Eletrônico do Paciente na forma de aplicativo para smartphones e tablets, voltado para estomatologia, tendo como parâmetro o prontuário físico utilizado na clínica escola, visando atender as suas necessidades como também sendo uma alternativa que possibilite aos docentes e discentes uma ferramenta que padronize o atendimento de forma organizada, sistematizada e ágil. Para o desenvolvimento do PEP algumas características foram consideradas como: agilidade, organização, redução de gastos, além de permitir novos recursos vinculados ao aprendizado do estudante, como uso de fotos ou uploads de arquivos vinculado ao paciente, como é citado por Silva (2019), no qual o uso de imagens como um novo modelo para a utilização de lâminas de microscopia no aprendizado prático, permitindo levar tais recursos coletados para discussões em sala de aula, assim como o uso de tecnologias moveis também pode permitir o estudo em ambientes cooperativos mesmo em locais ou momentos diferentes.

\section{Material e Métodos}

Tomando como base a ideia do Inquiry Based Learning (IBL) que segundo Santos (2018) se trata de uma metodologia que pode ser suportada com o uso de tecnologia, no qual o estudante por meio de uma abordagem construtivista deverá investigar um problema ou questão, exigindo um raciocínio baseado em evidências e soluções criativas. Ou seja, a criação do aplicativo de um prontuário eletrônico permitirá que o estudante no processo de anamnese realize uma investigação prática do seu paciente, sendo acompanhado pelo professor, permitindo ao final chegar a uma possível solução do problema.

O desenvolvimento do Estudo de Caso foi baseado em duas etapas: 
Desenvolvimento do aplicativo e sua validação.

\subsection{Desenvolvimento do Aplicativo}

Foi constituída uma equipe da pesquisa, composta por professores, alunos e funcionários dos cursos de odontologia e de análises de sistemas que participou ativamente da etapa de levantamento de requisitos, no qual foram observadas as necessidades dos stackholders. Foram examinados os prontuários físicos e POP's (Protocolos Operacionais Padrão) de funcionamento da clínica escola. O POP é um documento que contém todos os passos para a realização de uma atividade, e quem a executa, tendo como objetivo sistematizar as ações de assistência em saúde, dentro de princípios técnicos e científicos, bem como contribui para diminuir as distorções adquiridas na prática, tendo também finalidade educativa (WALTER et al., 2016). A partir destes documentos e da revista na literatura foram elaborados os protótipos das telas.

Para a definição de acesso ao aplicativo foram definidos dois níveis de usuário: Estudante - Está autorizado a realizar cadastro e condutas dos pacientes; Professor - Está autorizado a realizar, além do cadastro, edição de qualquer ato do aluno no aplicativo; como a evolução de condutas, permitindo fazer o processo avaliativo do aluno na clínica.

Para implementação do aplicativo foi utilizado o framework Ionic e Cordova. $\mathrm{O}$ Ionic ao contrário de um aplicativo nativo, é desenvolvido usando tecnologias web como JavaScript, HTML e CSS, e utiliza o Cordova para acessar recursos nativos como a câmera do smartphone, permitindo de forma mais prática a construção de um produto que esteja disponível em Android ou iOS. Para garantir a disponibilidade e segurança das informações dos pacientes, foi também desenvolvido um webservice. Por armazenar dados dos prontuários dos pacientes da clínica da instituição, este webservice está alocado dentro da própria estrutura da instituição.

Devido ao fato de as informações ficarem centralizadas em um webservice, o professor poderá acompanhar de seu smartphone no aplicativo (APP) em tempo real o trabalho realizado pelos estudantes. Para a construção deste webservice foi utilizado o interpretador NodeJS com framework Express, para recebimento das requisições e respostas do aplicativo. Os dados são salvos no webservice utilizando o Banco de Dados PostgreSQL.

Para realizar a comunicação entre o aplicativo e o servidor webservice, foi adotado como padrão o modelo Representational State Transfer ou REST, segundo Gruner et. al (2015), é uma arquitetura descentralizada que permite que sistemas de origens diferentes como sites e aplicativos possam compartilhar os mesmos dados com segurança, mantendo a interoperabilidade de plataformas sem dependências de linguagens de programação. $O$ sistema para adotar o padrão REST, ainda segue os métodos de envio de requisição GET, POST, PUT e DELETE através de URL's ao qual deve informar o recurso manipulado no webservice e requisições que funcionem de forma independentes, que ainda segundo os autores supracitados são propriedades usadas para considerar um webservice RESTful, ou seja, que contenha todas as propriedades da arquitetura.

O protótipo do PEP toma como base o workflow (fluxo de trabalho) presente na clínica atualmente. Para direcionar o usuário para seu módulo específico, será necessário que o mesmo se autentique no sistema através de uma tela de login.

Nesta tela, o usuário deverá informar um e-mail e senha cadastrada no sistema, para identificar-se. Inicialmente, esses dados são cadastrados diretamente no back-end, evitando que pessoas não autorizadas possam criar contas e acessarem o sistema. Após ter informado suas credenciais válidas, o aplicativo irá redirecionar o usuário para um módulo, podendo este ser o do estudante ou do professor. 
Para garantir a integridade dos dados no servidor, evitando que algum estudante com conhecimento em programação possa realizar um processo de engenharia reversa e modifique o comportamento do aplicativo enviando dados inválidos ou alterando notas no webservice, foi adotado como autenticação o modelo JWT. Ethelbert et al (2017) descreve Json Web Token (JWT) como uma estrutura de um token padronizado em três partes (Header, Payload e Signature) de informações armazenadas no formato JSON e compactadas em codificação de Base64. Através desse token é possível armazenar dados de identificação do solicitante, assim como garantir a que os dados do token não foram alterados de forma maliciosa.

Após a autenticação com sucesso, um token é retornado ao aplicativo, podendo identificar se o usuário é estudante ou professor. Este token fica salvo no próprio aplicativo e a cada requisição enviada ao servidor a uma rota com acesso restrito, o aplicativo deve enviá-lo também no headers da requisição.

As subseções a seguir demonstram com maior detalhe os recursos presentes nos módulos do estudante e professor com seus devidos recursos.

\subsubsection{Módulo do Estudante}

Com a sua credencial informada com sucesso, o estudante será redirecionado para a tela com a listagem de pacientes (Figura 1). A lista presente nessa tela, indicará apenas os pacientes cadastrados pelo próprio estudante, garantido que este não tenha acesso as informações dos demais paciente, garantindo assim o sigilo dos dados. À medida que novos pacientes chegam a clínica, o estudante poderá adicioná-los, clicando no ícone de adicionar ("+") na barra de ferramentas na parte superior da tela, como pode ser visto na Figura 1.

\begin{tabular}{l}
$\equiv \quad$ Pacientes \\
Q Digite o nome... \\
\hline Aline \\
Arthur \\
Carlos \\
Sonia \\
Thiago
\end{tabular}

Figura 1 - Listagem de pacientes

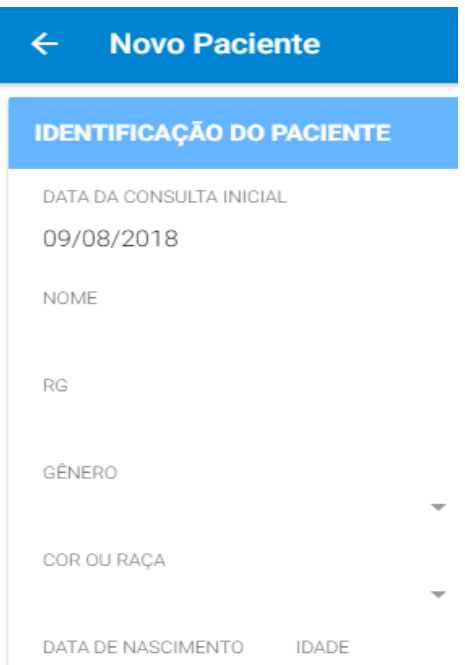

Figura 2 - Cadastro inicial do paciente

Todo paciente cadastrado pelo prontuário, estará vinculado a um estudante, de modo que, este poderá acompanhar seu paciente ao longo do tratamento. Ao solicitar um novo cadastro, o estudante tem o acesso a tela (Figura 2) onde acontece o cadastro de identificação do paciente. O termo de consentimento pode ser impresso e posteriormente assinado manualmente pelo paciente. Após o cadastro, o estudante ao clicar no seu paciente, será direcionado para uma nova tela (Figura 3), na qual poderá preencher os dados relacionados ao prontuário odontológico do paciente. 


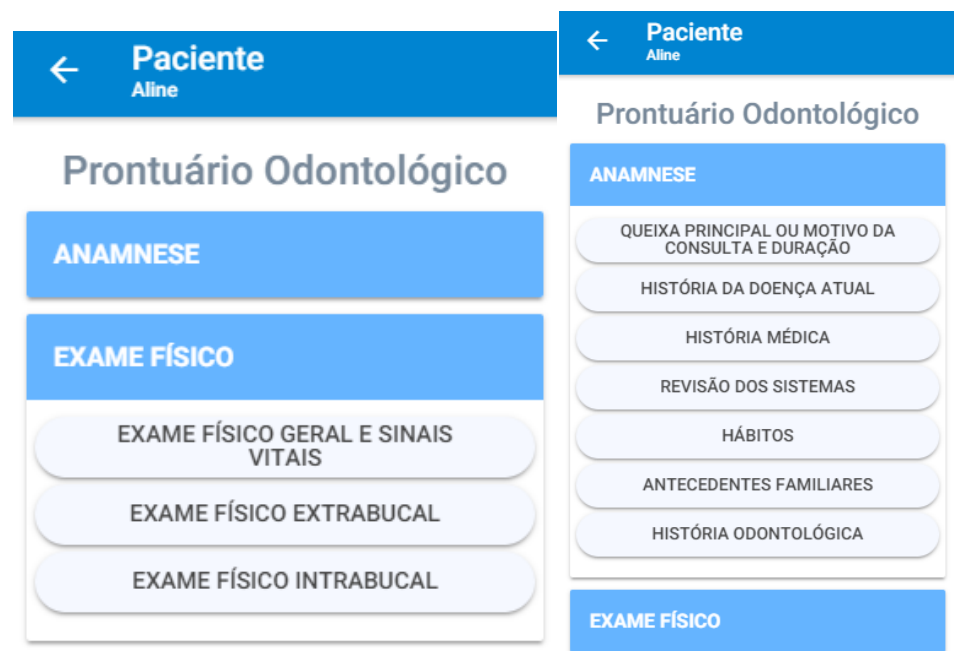

Figura 3 - Visualização do prontuário odontológico

Após preencher o prontuário odontológico, o estudante poderá também acompanhar e listar as condutas de seus pacientes, como pode ser observado na Figura 4, uma opção de realizar "conduta" estará disponível. Ao acessar esta opção, o estudante poderá ter acesso a todas as condutas por ele já realizadas, assim como também cadastrar nova conduta clicando no botão adicionar $(+)$ na barra de ferramentas no topo da tela (Figura 4 e 5). Assim que o conteúdo do paciente for preenchido e o estudante selecionar a opção "Salvar", este não poderá mais ser alterado. O processo de alterar uma informação cadastrada pelo estudante, deverá ser solicitado diretamente ao professor responsável que ao acessar seu módulo terá a permissão de editar os dados cadastrados pelo aluno. Figura 7.

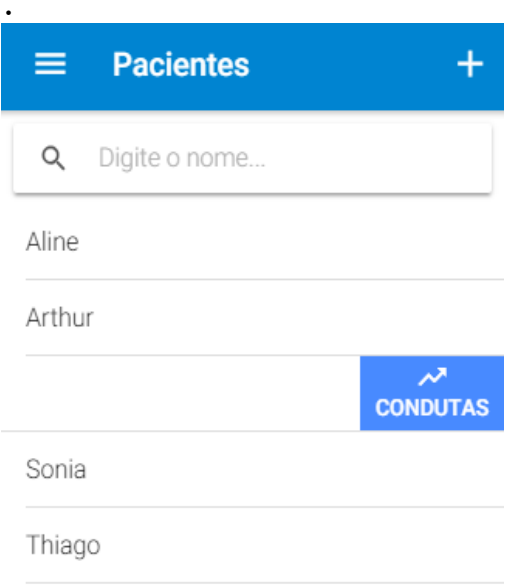

Figura 4 - Listagem dos pacientes e suas condutas

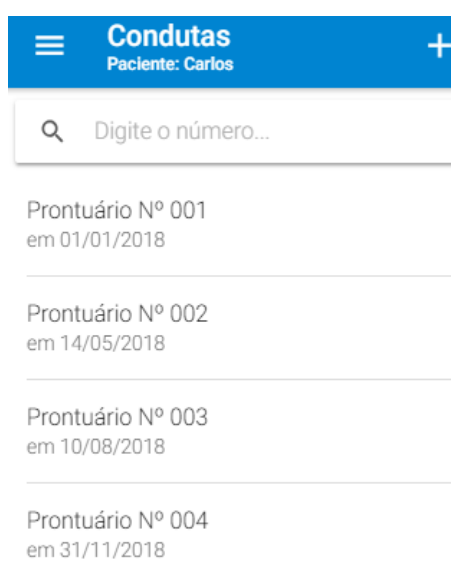

Figura 5 - Condutas odontológicas

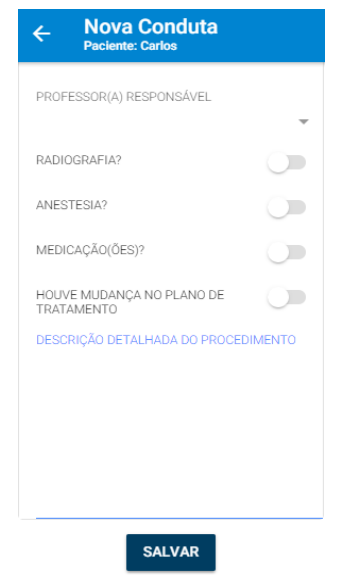

Figura 6 - Cadastro de nova conduta odontológica

Com o levantamento dos requisitos e necessidades apontadas pelos stakeholders durante o período de teste nos grupos focais, o aplicativo sofreu alterações para maior satisfação e conforto dos envolvidos. No módulo do estudante foram adicionados os formulários restantes como, avaliação dentária (Figura 7), acompanhamento de nota atribuída pelo professores ao estudante pela avaliação do paciente e impressão do prontuário em formato $\mathrm{PDF}$. 


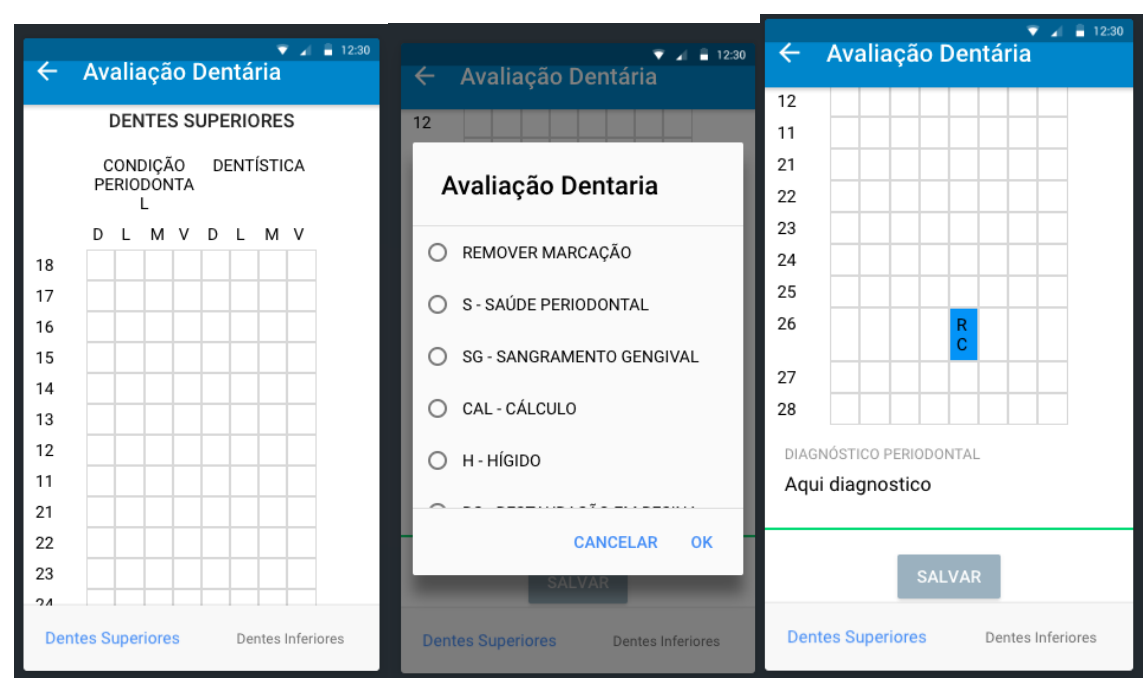

Figura 7 - Formulário de avaliação dentária

Para aumentar a segurança dos dados do paciente coletado no aplicativo pelos estudantes, sendo um dos requisitos levantados pelos stakeholders, foi adicionado o plugin "cordova-plugin-privacyscreen" no aplicativo, que consiste em evitar que um usuário possa tirar um printscreen na aplicação e repassar as informações para outra pessoa.

\subsubsection{Módulo do Professor}

O módulo do professor poderá ser acessado apenas pelos usuários que foram cadastrados no sistema como professor. Em vista disso, caso um usuário realizar uma autenticação na tela de login como professor, será redirecionado para a tela com a listagem de todos os estudantes com contas ativas no aplicativo.

Neste momento, o professor poderá selecionar um dos estudantes, no qual terá acesso a todos os seus pacientes, além de poder visualizar e editar qual dado cadastrado pelo estudante em relação ao paciente, inclusive os campos que estejam travados para os estudantes editarem, como os dados pessoais do paciente.
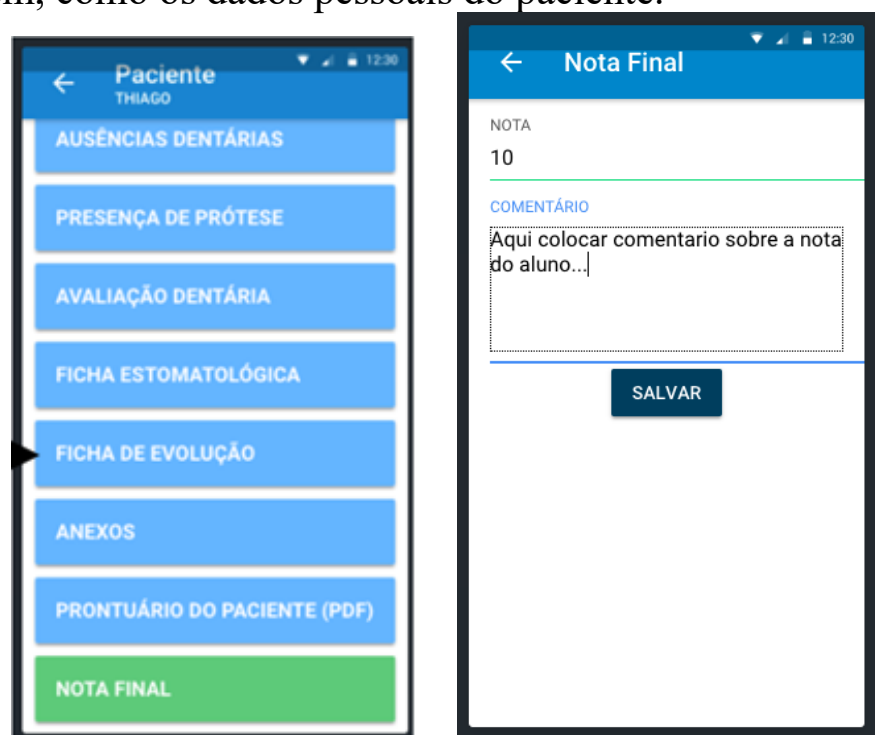

Figura 8 - Nota atribuída ao estudante

A existência da funcionalidade de perfil de professor e estudante foi reforçada, com a adição do recurso de atribuição de nota ao estudante por parte do professor. Este é um recurso o qual o professor poderá selecionar um dos pacientes de seus estudantes e na opção "Nota Final" informar a nota merecida ao estudante, assim como um comentário V. $17 \mathrm{~N}^{\circ}$ 3, dezembro, 2019 RENOTE DOI: 
justificando a nota (Figura 8), sendo essas informações apenas de acesso pelo próprio estudante e professor. Esta função permite uma interação maior entre professor e estudante, possibilitando um feedback no processo de aprendizagem do estudante, de modo que, o mesmo possa observar seus erros, passando por uma reflexão, depuração e aplicação de novas hipóteses.

Sobre a possibilidade de imprimir o formulário com os dados do paciente, fora disponibilizado no aplicativo a funcionalidade, que permite que seja gerado um arquivo no formato de PDF com todos os dados do paciente preenchidos (Figura 9).

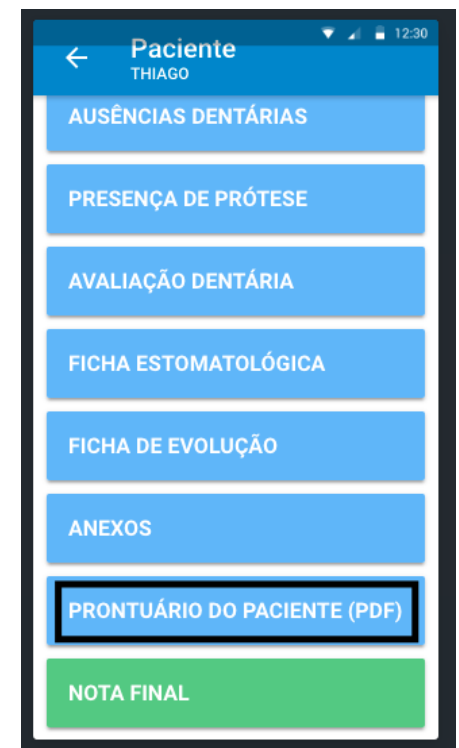

Figura 9 - Tela de impressão do prontuário em PDF

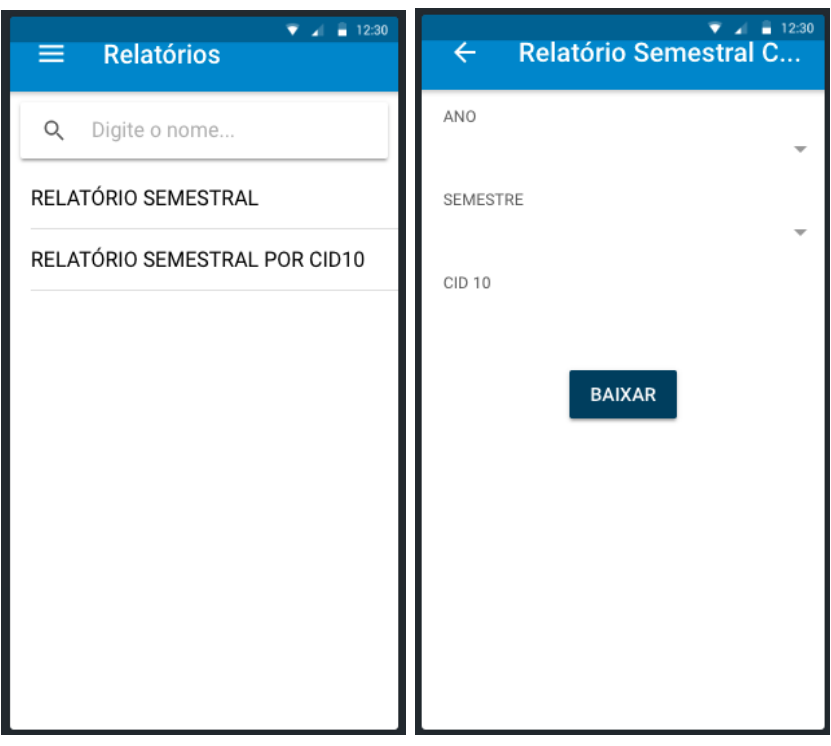

Figura 10 e 11 -Telas para gerar relatório Semestral ou por CID

Outro recurso que está sendo disponibilizado pelo aplicativo em sua nova versão, é voltado ao âmbito de pesquisas. O recurso consiste na criação de relatórios em arquivos no formato de PDF listando o total de pacientes cadastrados no semestre ou baseado no CID, classificando-os por: gênero; faixa etária; raça/cor; e escolaridade. Este é um recurso limitado apenas aos usuários com perfil de professor, uma vez que envolve informações todos os pacientes da clínica. Os acessos a esses relatórios podem ser vistos na Figura 10 e 11 .

\section{Validação do Software}

Para a fase de validação, 16 participantes foram recrutados respeitando os seguintes critérios de inclusão: ser coordenador da clínica integrada, docente da disciplina de estomatologia, radiologia e patologia bucal, docente da disciplina de desenvolvimento de aplicativos móveis do curso de sistema de informações, discentes de odontologia matriculados a partir do $4^{\circ}$ ao $10^{\circ}$ período da graduação de odontologia, ser discente do curso de sistema de informação, funcionário da instituição e fazer parte da equipe de secretaria do curso de odontologia. O projeto foi aprovado pelo Comitê de Ética e Pesquisa.

A pesquisa foi realizada em uma clínica escola de odontologia do estado de Alagoas.

Durante a fase de validação do software foi utilizado a metodologia de grupos focais e oficinas. Foram realizadas três oficinas. Duas delas envolvendo a idealização e o desenvolvimento do aplicativo e a final dentro da clínica escola de odontologia durante o atendimento dos pacientes, que é realizada pelos estudantes, para testar e avaliar a funcionalidade como também a aplicabilidade do software. 
A técnica de grupos focais é uma técnica de pesquisa qualitativa, derivada das entrevistas grupais que coletam informações por meio de integrações coletivas (BONFIM, 2009). As falas do grupo e das oficinas foram integralmente transcritas e analisadas através da análise temática.

Os roteiros de questão para o Grupo Focal foram formulados baseando-se na rotina do atendimento na clínica, que envolve as especialidades de estomatologia, radiologia e patologia bucal. Além das questões operacionais, foram avaliados o nível de compreensão e os sentidos dos envolvidos com esta mudança.

As questões norteadoras foram:

- O que entendemos por prontuário eletrônico?

- O que você acha de substituir o prontuário tradicional por prontuário eletrônico?

- Você acha que haverá vantagens em usar esta ferramenta na clínica?

- Você acha que existem vantagens desta informatização ser em forma de dispositivos móveis?

- Quais as dificuldades do uso do aplicativo e da sua implementação?

\subsection{Resultados e Discussão}

O surgimento de novas tecnologias possibilitou que os prontuários não fossem somente depositórios de informações (GODOY et al., 2011). Os arquivos digitais fornecem além do armazenamento de informações oferecem privacidade, praticidade e rápida atualização de dados entre outros (SOARES et al., 2006).

Diante disso, as falas dos participantes durante a abordagem do grupo focal enfatizaram que a substituição do prontuário físico pelo PEP possibilita melhor organização, fácil acessibilidade e economia de tempo.

$P 6$ - "Toda essa parte de logística e arquivamento do prontuário e a economia de tempo nesse sentido faz você acessar mais rápido as informações".

P1- "[...] para facilitar todo o funcionamento das clínicas eu acho que o PEP vem para colaborar".

As falas acima enfatizam as vantagens da logística do PEP, a organização mais sistematizada dos dados dos pacientes, bem como a possibilidade do acesso rápido as informações uma vez que todas essas vantagens colaboram para o melhor funcionamento da clínica escola. Para Ribeiro et al., (2018), quando os dados dos pacientes são arquivados de maneira organizada, é possível o compartilhamento das informações entre diferentes profissionais de saúde, uma vez que o uso do prontuário eletrônico promove a integração dos dados e favorece maior controle da continuidade do atendimento e acompanhamento do paciente. Nesse mesmo pensamento a fala de P2 relata expectativa na inclusão dos exames de imagens tornando o histórico do paciente acessível e integrado.

P2- "[...] a expectativa é que o PEP realmente abrace todas essas informações inclusive os exames de imagens [...]".

O prontuário vai além de dar suporte à assistência ao paciente, ele também é útil para tomada de decisão, é fonte de informação a ser compartilhada entre os profissionais de saúde, favorece o suporte a pesquisa (clínica, estudos epidemiológicos) sendo também, considerado um documento legal (JENAL; EVÓRA, 2012). A tecnologia móvel é uma realidade crescente na sociedade, influenciando um novo perfil de entrega de informações e interatividade com seus usuários. A principal característica dos aplicativos mobile é a facilidade de manejo e operação. (SOUZA et al., 2013).

Sobre os benefícios do uso do PEP em dispositivos mobile as falas de P2 cita a limitação do prontuário convencional destacando a mobilidade e facilidade de acesso as informações.

P2- "Então hoje o prontuário fisico têm suas limitações, e o app é só abrir[...]”. 
A fala de P2 parte da mesma idéia de Tibes, Dias e Mascarenhas (2014) que explica que a característica principal dos aplicativos mobile é a quebra da limitação da mobilidade, uma vez que os smartphones são como um computador de bolso e podem acompanhar seu usuário 24 horas por dia onde ele estiver. Porém, alguns participantes apontaram algumas desvantagens que podem surgir com o uso dos aplicativos móveis. Dentre as desvantagens relatadas destacou-se a questão da segurança dos dados dos pacientes, a dependência de internet, e de não poder utilizar o dispositivo móvel offline

P3 - "Essa parte da segurança eu acho um ponto bem crucial [...]é isto que eu estou falando, a minha preocupação é com as imagens".

P3 enfatiza uma preocupação com a segurança das informações contidas no PEP, no caso dos arquivos digitais essa é uma preocupação de extrema relevância de acordo com a literatura, porém o levantamento deste tema não estava previsto pela equipe de pesquisa. As referidas falas no quesito segurança corroboram com a literatura uma vez que as informações dispostas no PEP estão de certo modo vulneráveis comprometendo o sigilo legal dos prontuários (RONDINA; CÂNEO; CAMPOS, 2015).

Após o levantamento das questões de segurança e demais questionamentos, os pesquisadores realizaram todas as modificações até a versão final apresentada na seção anterior, adicionando

\section{Conclusão}

Conclui-se nesse estudo a implementação do PEP na clínica escola de odontologia trará agilidade, segurança, maior capacidade de armazenamento uma vez que o sistema foi considerado completo e adequado à rotina na clínica escola. Este estudo demonstrou importância da participação colaborativa grupal na busca das melhorias e na qualidade no desenvolvimento do software, assim como a necessidade de uma constante interação dos envolvidos para uma melhor qualidade do registro das informações.

Por fim, o PEP se mostrou uma ferramenta eficaz com designer autoexplicativo, didático que permitiu o acesso remoto seguro e fácil, possibilitando registrar e armazenar fotos e outras informações detalhadas dos pacientes e de suas lesões, sendo uma excelente opção para a utilização em clinicas escolas.

\section{Referências}

BONFIM, L. A. Grupos focais: conceitos, procedimentos e reflexões baseadas em experiências com o uso da técnica em pesquisas de saúde. Physis Revista de Saúde Coletiva, v. 19, n. 3, p. 777-796, 2009.

BRAGA, R. D.; MONTEIRO, A. L. N.; MOTA, F. C. S.; LUCENA, F. N.; ROTTA, R. F. R. Validação do prontuário eletrônico do paciente em uma instituição de ensino superior em saúde: relato da experiência no módulo anamnese. Journal of Health Informatics, v. 5, n.1, p. 30-35, 2013.

DUFTSCHMID, G.; WRBA, T.; GALL, W.; DORDA, W. The strategic approach of managing healthcare data exchange in Austria. Methods Inf Med . p. 124-132, 2004.

ETHELBERT, O., MOGHADDAM, F. F., WIEDER, P., \& YAHYAPOUR, R. A JSON Token-Based Authentication and Access Management Schema for Cloud SaaS Applications. 2017 IEEE 5th International Conference on Future Internet of Things and Cloud (FiCloud). doi:10.1109/ficloud.2017.29 
GRUNER, S., PFROMMER, J., \& PALM, F. RESTful Industrial Communication With OPC UA. 2016. IEEE Transactions on Industrial Informatics, vol. 12, n.5, 18321841. doi:10.1109/tii.2016.2530404

GODOY, J.S.M.; GONÇALVES, L.S.; PERES, A.M.; WOLF, L.D.G. O uso do prontuário eletrônico por enfermeiros em Unidades Básicas de saúde brasileiras. J. Health Inform, v. 4, n.1, p. 3-9. 2011.

JENAL, S.; ÉVORA, Y.D.M. Implantação de Prontuário Eletrônico do Paciente. Rev.J. Health Inform, v. 4, n. 4, p.176-81, 2012.

MACHADO, R. P. A.; SHERMA, A. P.; PISA, I. T. Uso da informática na odontologia. ClipOdonto, v.4, n. 1, p.31-37, 2012.

RONDINA,J. M.; CÂNEO, P.K.; CAMPOS, M.S.; RODRIGUES, Y.P. Prontuário eletrônico do paciente: comparação de um hospital de ensino com outras instituições de saúde. Rev.Saúd.Digi.Tec.Edu, Fortaleza, v. 2, n.3, p.41-50, 2017.

SANTOS, M.C.M.; PACHECO, T.C.L; Lesões do complexo maxilofacial: estudo de prevalência e construção de uma ferramenta informatizada para armazenamento e recuperação de dados (software) para avaliação das lesões. 2010. Trabalho de conclusão de curso em odontologia (Graduação em Odontologia) - Centro Universitário Cesmac, Maceió, Alagoas, 2010.

SANTOS, R. S. R. dos. O Simulador Photolab num Espaço de Aprendizagem por Investigação no Desenvolvimento de Competências do Processo Científico. Relatório de Projeto (Mestrado em Utilização Pedagógica das TIC) Escola Superior de Educação e Ciências Sociais. Leiria, Portugal. 2018.

SARAIVA, A.S. A importância do prontuário odontológico com ênfase nos documentos digitais. Rev. bras. odontol. Rio de Janeiro. v. 68, n. 2, p. 157-60, 2011.

SILVA, P. F. da. O uso das Tecnologias Digitais como Ferramentas Cognitivas. RENOTE, v.17, n.2, 2019. DOI: 10.22456/1679-1916.96588

SOARES, M.G.; MORAES, M. E. L; MORAES, L. C.; FILHO, E. M.; CASTILHO, J. C. M.; TAKESHITA, W. M. Arquivos Digitais em Odontologia. v. 60, n.4, p. 277 $280,2006$.

SOUZA, R. C; ALVES, L. A. C.; ALVES, A. C.; HADDAD, A. E.; MACEDO, M. C. S.; CIAMPONI, A. L. Processo de criação de um aplicativo móvel na área de odontologia para pacientes com necessidades especias. Rev. Abeno. vol 13. Londrina, 2013.

TIBES, C. M. S.; DIAS, J. D.; MASCARENHAS, S. H. Z. Aplicativos móveis desenvolvidos para a área da saúde no Brasil: Revisão integrativa da Literatura. Rev Min Enferm. v.18, n. 2, p. 472-478, 2014.

WALTER, R.R.; GEHLEN, M.H.; ILHA. S; et al. Standard operating procedure in the hospital context: the nurses' perception. RevFundCare.Online, v. 8, n .4,p. 5095- 5100, 2016. 